\title{
Amaurose bilateral irreversível pós-cirurgia cardíaca
}

\author{
Irreversible bilateral amaurosis after heart surgery
}

Valdir Cesarino de SOUZA ${ }^{1}$, Alex BARBOSA ${ }^{1}$, Basílio Serrano de SOUSA FILHO ${ }^{1}$, Kleber Oliveira de SOUZA ${ }^{2}$

RBCCV 44205-701

\section{Resumo}

Paciente do sexo feminino, 16 anos, portadora de cardiopatia congênita, tipo comunicação interatrial. Submetida a correção cirúrgica, evoluiu nas primeiras horas com sangramento e instabilidade do quadro hemodinâmico. Corrigidas tais complicações, evoluiu com perda progressiva e bilateral da visão. Após as primeiras horas de pós-operatório, apresentava acentuado edema de papilas ópticas, além de hipertensão intra-ocular. No transcorrer de 21 dias, evidenciavam-se sinais de atrofia dos nervos ópticos com instalação, bilateral e irreversível, de amaurose, apesar das condutas terapêuticas instituídas. No seguimento de dois anos, a paciente evoluiu com cegueira bilateral e lesão óptica irreversível.

Descritores: Amaurose. Cegueira. Procedimentos cirúrgicos cardíacos, efeitos adversos. Complicações pós-operatórias. Doenças do nervo óptico, etiologia.

\begin{abstract}
A 16-year-old female patient, with a congenital heart defect (interatrial communication) underwent heart surgery. She evolved with complications in the immediate postoperative period with sudden hemorrhagic hypotension and progressive loss of sight in both eyes. In the postoperative period there was a high intraocular pressure and optic disk edema. Optic atrophy and irreversible bilateral blindness was confirmed 21 days after heart surgery despite treatment. In two years of follow-up, the patient is completely blind with irreversible optic atrophy
\end{abstract}

Descriptors: Amaurosis. Blindness. Cardiac surgical procedures, adverse effects. Postoperative complications. Optic nerve diseases, etiology.

Trabalho realizado no Instituto de Cirurgia Cardiovascular da Paraíba (ICCP)

Rua Siqueira Campos, 543 - CEP 58108-540 - Campina Grande, PB, Brasil

e Faculdade de Medicina da Universidade Federal de Campina Grande (UFCG)

1 - Professores da Faculdade de Medicina da UFCG, Cirurgiões Cardiovasculares do ICCP.

2 - Acadêmico da Faculdade de Medicina da UFCG

Endereço para correspondência: Valdir Cesarino de Souza. Rua Siqueira Campos, 543 - CEP 58.108-540 - Campina Grande, PB, Brasil Fone: (83) 3413640. Fax: (83) 3414132

E-mail: valdir.souza@sbccv.org.br 


\section{INTRODUÇ̃̃O}

A perda total ou parcial da visão pós-correção de defeitos cardíacos sob circulação extracorpórea (CEC) é rara e pouco elucidada. Sua incidência varia de $0,1 \%$ a $1 \%$ [1-4]. Porém, quando tal complicação se torna irreversível, sua incidência ainda é mais rara, 0,04\% [2]. Embora tal alteração visual seja resultante de diversos fatores, a mesma está freqüentemente associada a sangramentos cirúrgicos com queda acentuada da pressão arterial sistêmica, tempo elevado de CEC, microembolias, entre outras [4,5]. Seu tratamento é bastante controverso segundo a literatura, contudo a precocidade com que é feito o diagnóstico é essencial para um bom prognóstico [4].

O objetivo deste trabalho é relatar um caso de perda bilateral e irreversível da visão pós-correção de uma cardiopatia congênita.

\section{RELATO DE CASO}

Paciente do gênero feminino, 16 anos de idade, normolínea, encaminhada ao serviço de cardiologia com queixas freqüentes de palpitações e cansaço aos médios esforços, com piora progressiva, sobretudo nos últimos meses.

Ao exame físico apresentava-se em regular estado geral, afebril, acianótica, levemente dispnéica. À ausculta cardíaca denotava-se o ritmo cardíaco irregular, segunda bulha desdobrada, com freqüência de 92 b.p.m. e leve sopro sistólico em foco pulmonar. A pressão arterial era de 110 x 70 $\mathrm{mmHg}$; o restante da avaliação semiológica, normal.

O exame radiológico evidenciava leve aumento da área cardíaca às custas do ventrículo direito, enquanto que o eletrocardiograma apresentava ritmo sinusal com algumas extrassístoles supraventriculares, além de distúrbio da condução do ramo direito.

Encaminhada ao setor de ecocardiografia e, posteriormente, ao serviço de hemodinâmica, constatou-se uma má formação cardíaca acianótica, com hiperfluxo pulmonar, tipo comunicação interatrial (CIA), localizada ao nível da fossa oval, com cerca de $2,5 \mathrm{~cm}$ de diâmetro, e pressões em câmaras direitas levemente aumentadas. Após análise dos dados, foi recomendado o tratamento cirúrgico.

$\mathrm{O}$ ato operatório realizou-se sob anestesia geral e toracotomia mediana transesternal. Após estabelecimento da CEC, realizou-se atriotomia direita. ACIA foi visualizada conforme indicavam os exames e a mesma foi ocluída com retalho de pericárdio bovino. $\mathrm{O}$ átrio foi suturado, a perfusão extracorpórea cessada e o restante da operação proferida conforme o habitual. Tanto o ato operatório como o anestésico transcorreu dentro da normalidade. $\mathrm{O}$ tempo de CEC foi de 32 minutos e o de anóxia de 26 minutos.

Durante as três primeiras horas de pós-operatório, verificou-se que a paciente apresentava sangramento insidioso (cerca de $6 \mathrm{ml} / \mathrm{kg}$ de peso). Optou-se inicialmente por correções dos distúrbios de coagulação pertinentes ao ato operatório, além da reposição volêmica e uso de drogas vasopressoras. Como o sangramento persistiu, desenvolveu-se instabilidade do quadro hemodinâmico com hipotensão acentuada e choque hipovolêmico. Diante do quadro, a revisão hemostática foi desencadeada sendo suturada uma tributária da veia braquiocefálica esquerda; tal procedimento transcorreu sem alterações significativas.

Estabilizado o quadro cirúrgico, pós-correção operatória do sangramento, a paciente retornou ao centro de tratamento intensivo, estável hemodinamicamente, sem necessidade de novas transfusões e/ou drogas vasoativas.

Poucas horas após a extubação, a doente referiu embaçamento visual bilateral com a perda progressiva da visão e fortes dores em ambos os globos oculares. Solicitou-se o parecer de um especialista, o qual realizou fundoscopia bilateral evidenciando edema acentuado dos discos ópticos, e tonometria, denotando aumento da pressão intra-ocular. Diante desses dados, iniciou-se o uso de corticóide e diurético osmótico (manitol). Mesmo com a terapia instituída, o que se observou foi a perda progressiva da visão.

Além dos exames oftalmológicos habituais, foram realizados: tomografia computadorizada, ressonância magnética, ultra-sonografia ocular, dentre outros, que constataram, 21 dias após a correção da cardiopatia congênita, atrofia bilateral irreversível dos nervos ópticos conseqüente à isquemia posterior.

Dois anos após o desencadeamento dessa complicação cirúrgica, novos exames foram realizados confirmando mais uma vez a atrofia acentuada bilateral de ambos nervos ópticos e amaurose bilateral definitiva.

\section{COMENTÁRIOS}

Apesar de rara, a perda da visão pós-cirurgia cardíaca é bem documentada e sua incidência varia de $0,1 \%$ a $1 \%$ [1-4]. No entanto, dados da literatura concluem que a incidência de amaurose bilateral definitiva é cerca de $0,04 \%$ [2].

A vascularização do nervo óptico difere de acordo com sua localização: a porção anterior é basicamente vascularizada pelos ramos distais das artérias ciliares posteriores e a porção posterior pela artéria central da retina, local este onde o nervo óptico é mais vulnerável à isquemia, sendo, portanto, a região mais freqüentemente acometida por esta alteração, sobretudo naqueles pacientes que desenvolvem hemorragias associadas à hipotensão póscirúrgica. Por conseguinte, a amaurose resultante da isquemia do nervo óptico varia de acordo com a instalação da lesão, podendo ser classificada em neuropatia anterior e posterior [4]. 
Algumas das causas relatadas na literatura para a perda visual pós-cirurgia cardíaca incluem: isquemia anterior ou posterior do nervo óptico, lesões corticais, trombose arterial e/ou venosa retiniana, entre outras $[4,5]$.

Os principais fatores de risco para o desencadeamento de neuropatia óptica pós-cirurgia cardíaca são: paciente portador de glaucoma ou outro problema oftalmológico, cirurgia com tempo de CEC prolongado, excessiva hemodiluição, hipotermia sistêmica prolongada, sangramento pós-operatório acentuado e a necessidade de medicações vasoconstrictoras, que predispõem à lesão do nervo óptico $[3,6]$.

Diversos autores chamam atenção para o fato da isquemia ser decorrente principalmente de hipotensão arterial, hipotermia sistêmica prolongada e ativação de certos complementos durante a CEC, como angiotensina e serotonina [2,5].

A suspeita da isquemia do nervo óptico é dada quando o paciente relata perda visual, total ou parcial. Seu prognóstico depende da precocidade com que se faz o diagnóstico, além da extensão e localização da isquemia [4]. Vários exames são preconizados para o esclarecimento dessa complicação óptica, desde o exame oftalmoscópico direto até outros como a ressonância magnética e a tomografia computadorizada [4].

Existem várias divergências entre autores no que se refere ao tratamento da cegueira como conseqüência de cirurgia cardíaca, enquanto alguns [4] preconizam o uso de altas doses de esteróides, antiagregantes plaquetários, inibidores da anidrase carbônica e operação para descompressão do nervo óptico; outros [6] concluíram que nenhum desses procedimentos se comprovou eficaz no tratamento dessa complicação, sobretudo no que se refere à fenestração do nervo óptico [3,6]. Contudo, estas podem ser consideradas medidas profiláticas que devem ser tomadas para evitar a isquemia e lesão irreversível do nervo óptico [6].

\section{CONCLUSÃO}

Instalada a amaurose pós-cirurgia cardíaca, o índice de cura é bastante baixo, sobretudo se as medidas profiláticas e as condutas terapêuticas não forem observadas. Mesmo com tais procedimentos, torna-se quase impossível para as equipes preverem o desencadeamento desta temida complicação, principalmente devido a transtornos inerentes ao ato cirúrgico e às variações anatômicas existentes na vascularização do globo ocular.

\section{REFERÊNCIAS BIBLIOGRÁFICAS}

1. Shahian DM, Speert PK. Symptomatic visual deficits after open heart operations. Ann Thorac Surg 1989;48:275-9.

2. Sweeney PJ, Breuer AC, Selhorst JB, Waybright EA, Furlan $\mathrm{AJ}$, Lederman RJ et al. Ischemic optic neuropathy: a complication of cardiopulmonary bypass surgery. Neurology 1982;32:560-2.

3. Shapira OM. Kimmel WA, Lindsey PS, Shahian DM. Anterior ischemic optic neuropathy after open heart operations. Ann Thorac Surg 1996;61:660-6.

4. Williams EI, Hart JrWM, Tempelhoff R. Postoperative ischemic optic neuropathy. Anesth Analg 1995;80:1018-29.

5. Roth S, Barach P. Postoperative visual loss: still no answers yet. Anesthesiology 2001;95:575-7.

6. Busch T, Sirbu H, Aleksic I, Stamm C, Zenker D, Dalichau H. Anterior ischemic optic neuropathy: a complication after extracorporal circulation. Ann Thorac Cardiovasc Surg 1998;4:354-8. 